\title{
Corrigendum: Association between TAP1 gene polymorphisms and alopecia areata in a Korean population - Genet. Mol. Res. 14 (4): 18820-18827
}

Published online: December 28, 2015 (DOI: 10.4238/2015.December.28.31)

Corrected after publication: March 4, 2016 (DOI: 10.4238/gmr.150170751)

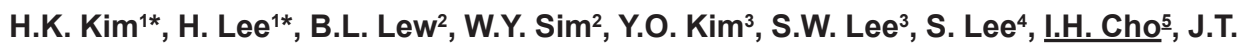
Kwon ${ }^{1}$ and H.J. Kim ${ }^{1,6}$

${ }^{5}$ Department of Convergence Medical Science, Brain Korea 21 Plus Program, and Institute of Korean Medicine, College of Korean Medicine, Kyung Hee University, Seoul, Republic of Korea 